\title{
Zika virus infection in semen: a call to action and research
}

\author{
Ayresleigh Rowland ${ }^{1}$ • Chantel I. Washington ${ }^{2}$ - Jeanne S. Sheffield ${ }^{3}$ • \\ Carlos A. Pardo-Villamizar ${ }^{4}$ • James H. Segars ${ }^{2}$ (D)
}

Received: 19 February 2016 / Accepted: 23 February 2016 / Published online: 5 March 2016

(C) Springer Science+Business Media New York 2016

The emergence of the Zika virus (ZIKV) epidemic in South and Central America has quickly become an international public health crisis. ZIKV often causes a self-limited febrile illness, but acute infection can be considerably more pernicious, leading to complications such as encephalitis, myelitis, and in some cases Guillain-Barré syndrome, which has led to a number of fatalities [1]. Furthermore, infection in pregnant women has been associated with severe teratogenic effects, including microcephaly and retinal lesions. The link between perinatal infections and microcephaly has prompted many local health officials to recommend that women delay pregnancy indefinitely [2].

Capsule Zika virus infection has far reaching implications for the field of assisted reproductive technologies. Zika virus has been isolated from semen and sexual transmission has been confirmed. It is incumbent on the field of reproductive sciences to lead the research efforts to enhance understanding and management of Zika virus infection as it relates to reproductive health and conception.

Ayresleigh Rowland and Chantel I. Washington are co-first authors.

James H. Segars

jsegars2@jhmi.edu

1 The Johns Hopkins University School of Medicine, 733 North Broadway, Baltimore, MD 21205, USA

2 Division of Reproductive Sciences and Women's Health Research, Department of Gynecology and Obstetrics, The Johns Hopkins University School of Medicine, 720 Rutland Avenue, Room 624 Ross Building, Baltimore, MD 21205, USA

3 Division of Maternal-Fetal Medicine, Department of Gynecology and Obstetrics, The Johns Hopkins University School of Medicine, 600 North Wolfe Street, Phipps Building, Room 203, Baltimore, MD 21287, USA

4 Department of Neurology, The Johns Hopkins University School of Medicine, David H. Koch Cancer Research Building II, 1550 Orleans Street, Room 1M16, Baltimore, MD 21287, USA
Zika virus is a mosquito-borne flavivirus, primarily transmitted by the Aedes aegypti mosquito [3]. The Aedes genus has a domestic predilection, preferring to live indoors and feed on humans [4]. Given these behaviors, it is not surprising that the geographic distribution of $A$. aegypti has closely mirrored human migration and urbanization [3]. The ZIKV was first isolated in Africa in 1947, where it was associated with sporadic cases. However, in recent years, other outbreaks occurred in the South Pacific. The first significant outbreak of ZIKV occurred on Yap Island, Micronesia, in 2007, followed by an epidemic in French Polynesia in 2013. Then, in 2015, the virus began to spread throughout the western hemisphere following the path of other arboviruses like dengue and chikungunya, starting with an outbreak in Brazil linked to the 2014 FIFA World Cup [5]. More than 20 countries in the Americas are now affected [6]. The temporal association of the ZIKV outbreak in Brazil with the marked increased incidence of microcephaly and other neurologic conditions has garnered worldwide attention. On February 1, 2016, the Director-General of the World Health Organization declared the epidemic a Public Health Emergency of International Concern, urging increased surveillance and mosquito population control. Phylogenetic analysis has revealed two main lineages of ZIKV, the African and the Asian lineages. The dominant viral strain currently circulating throughout South and Central America is closely related to the Asian lineage that previously affected French Polynesia, where it was associated with a similar rise in neurologic sequelae in newborns [7]. The teratogenic effects observed have begged the question of whether the virus has undergone a recombination event or alternative modification increasing its virulence.

There is an increasing volume of evidence that ZIKV is transmitted not only by mosquitos but also sexually, making the ZIKV the only arbovirus linked to sexual transmission [8]. The first reported case of male-to-female sexual transmission 
took place in Colorado in 2008 between a man returning from a trip to Senegal and his wife [8]. In addition to rash and arthralgias, he developed aphthous ulcers, prostatitis, and hematospermia; his wife, who had not left the country in one year, experienced similar viral symptoms two weeks after engaging in unprotected sexual intercourse. In January 2016, a second case of sexual transmission was reported in Dallas County, TX, in which a man returning from Venezuela passed the virus to his partner with no recent travel history [9]. The sexual transmission of ZIKV is further supported by reports showing the presence of viral RNA in the semen of infected men. Musso et al. [10] describe the case of a patient presenting with hematospermia ten weeks after the onset of Zika-like symptoms whose semen and urine samples tested positive for ZIKV by real-time reverse transcription PCR (rRT-PCR) and genome sequencing, though two sequential blood samples were negative. Additionally, a study by Atkinson et al. [11] reported detection of ZIKV RNA in semen up to 62 days after the illness onset - and at levels even higher than those in serum during the acute phase. Thus, these four reports provide evidence that ZIKV may be spread not only via a mosquito vector but also from person to person through sexual contact. Currently, all reported cases are male to female, but the possibility of female-to-male transmission remains. In addition to the human observations, an animal model of ZIKV infection developed in immunodeficient mice showed evidence of semen and testis infection, providing evidence that ZIKV is gonadotropic to the male reproductive tract.

The range of the Aedes mosquito is geographically determined, and thus, mitigation of the teratogenic risk for endemic areas will require development of vaccines or strategies to combat mosquito-borne illness. For regions of the world where the Aedes mosquito is not endemic, an important consideration is that sexual transmission represents the greatest risk for transmission of ZIKV.

The presence and persistence of ZIKV in semen raises several important questions. First, what is the true prevalence of ZIKV infection in semen? Serologic tests will likely lead to underdiagnosis, while semen analysis may prove a more accurate means of estimation; but semen samples are difficult to obtain. RT-PCR testing of urine samples from men have remained positive after acute infection has resolved, raising the possibility that the male urogenital tract may serve as a repository for the virus. Could penile swabs, as used for other STIs, be used to assess infection of the male reproductive tract? No studies to date have correlated this method with semen testing, but the idea seems to have merit. For women, testing with cervical swabs also might be of interest, even though female-to-male transmission has not been reported.

Second, for how long does semen remain infectious? This is a crucial question, since without information on the clearance of viral particles from the male reproductive tract, it is impossible to make evidence-based recommendations on the management of the male with ZIKV. A related question is whether fetal anomalies associated with ZIKV are equally likely with infection from the genital tract in the female, or mosquito-borne infection? It is possible that the viral load presented by semen to the pregnant cervix and female reproductive tract could lead to differential likelihood of fetal inoculation.

Third, after the acute illness passes, are there lasting effects on the testis and reproductive function in the male? Symptoms of prostatitis have been reported in men with ZIKV-infected semen. However, it is not currently known if orchitis occurs following ZIKV, or whether the reproductive tract is breached elsewhere. It seems well established that the virus can invade the nascent fetal blood-brain barrier, but it is not known whether the virus can breach the blood-testis barrier. If, as studies suggest, ZIKV may be gonadotropic in men, it is possible that infection of the adult might lead to long-term effects on sperm production in the male. Furthermore, perinatal infection might adversely affect gonadal development in male fetuses.

The implications of these questions are far reaching, but are of particular concern to the field of assisted reproductive technologies. As the epidemic develops in Latin America, it is possible that sperm banks in endemic areas could have samples that are infected. To assess this possibility, a study of sperm bank samples before and after the epidemic in endemic areas would provide useful information. Similarly, longitudinal studies of penile swabs of men in endemic areas that are positive for ZIKV serologically would be of interest to determine the pathogenicity of the virus for the male reproductive tract.

For sperm banks in areas that are not endemic, the important questions are how to prevent transmission of ZIKV from male samples and what, if any, testing should be done. Just as the American Red Cross has asked its blood donors who have recently traveled to Mexico, the Caribbean, or Central or South America to postpone blood donation until 28 days following their return to the continental USA, it would be prudent for sperm banks to institute similar precautions. For men who have traveled to endemic areas, the presence of hematospermia should raise concern regarding possible ZIKV infection. Additionally, semen samples from men who have recently seroconverted for ZIKV should not be used for ART, although it is not clear how long the semen remains infectious in such cases. A simple and cost-effective screening test is needed, but while RT-PCR works, the test is not widely available. Clearly, there is an urgent need for further research and, more importantly, there is a need for action. We must work together to better understand and prevent the lasting consequences of ZIKV on the reproductive tracts of both infected men and male fetuses of infected mothers. Only with this information will the field be equipped to manage infections and safeguard families using ART from undue suffering. 


\section{References}

1. Hayes EB. Zika virus outside Africa. Emerg Infect Dis. 2009;15: 1347-50.

2. Enfissi A, Codrington J, Roosblad J, Kazanji M, Rousset D. Zika virus genome from the Americas. Lancet. 2016;387:227-8.

3. Calvez E, Guillaumot L, Millet L, Marie J, Bossin H, Rama V, et al. Genetic diversity and phylogeny of Aedes aegypti, the main arbovirus vector in the Pacific. PLoS Negl Trop Dis. 2016. doi:10.1371/ journal.pntd.0004374.

4. Centers for Disease Control and Prevention. Surveillance and control of Aedes aegypti and Aedes albopictus in the United States. 2015. http://www.cdc.gov/chikungunya/resources/vector-control. html. Accessed 15 Jan 2016.

5. Gautret P, Simon F. Dengue, chikungunya and Zika and mass gatherings: what happened in Brazil, 2014. Travel Med Infect Dis. 2015. doi:10.1016/j.tmaid.2015.12.004.
6. Lucey DR, Gostin LO. The emerging Zika pandemic: enhancing preparedness. JAMA. 2016. doi:10.1001/jama.2016.0904.

7. Hynes NA. Zika virus. In: The Johns Hopkins POC-IT ABX guide. 2016. http://www.hopkinsguides.com/hopkins/view/Johns Hopkins_ABX_Guide/540658/all/Zika\%20virus. Accessed 4 Fē 2016

8. Foy BD, Kobylinski KC, Chilson FJL, Blitvich BJ, da Rosa A T, Haddow $A D$, et al. Probable non-vector-borne transmission of Zika virus, Colorado, USA. Emerg Infect Dis. 2011;17: $880-2$.

9. McCarthy M. Zika virus was transmitted by sexual contact in Texas, health officials report. BMJ. 2016. doi:10.1136/bmj.i720.

10. Musso D, Roche C, Robin E, Nhan T, Teissier A, Cao-Lormeau VM. Potential sexual transmission of Zika virus. Emerg Infect Dis. 2015;21:359-61.

11. Atkinson B, Hearn P, Afrough B, Lumley S, Carter D, Aarons EJ, et al. Detection of Zika virus in semen [letter]. Emerg Infect Dis. 2016. doi:10.3201/eid2205.160107. 